\title{
BRAF mutant metastatic colorectal cancers: new arrows in our quiver
}

\author{
Chiara Cremolini $^{1,2}$, Roberto Moretto ${ }^{1,2}$, Gemma Zucchelli $^{1,2}$, Alfredo Falcone ${ }^{1,2}$ \\ ${ }^{1}$ Unit of Medical Oncology 2, Azienda Ospedaliero-Universitaria Pisana, Pisa, Italy; ${ }^{2}$ Department of Translational Research and New Technologies \\ in Medicine and Surgery, University of Pisa, Pisa, Italy \\ Correspondence to: Chiara Cremolini, MD, PhD. Unit of Medical Oncology 2, Azienda Ospedaliero-Universitaria Pisana; Department of Translational \\ Research and New Technologies in Medicine and Surgery, University of Pisa, Via Roma, 67, 56126 Pisa, Italy. Email: chiaracremolini@gmail.com. \\ Comment on: Van Cutsem E, Huijberts S, Grothey A, et al. Binimetinib, Encorafenib, and Cetuximab Triplet Therapy for Patients With BRAF \\ V600E-Mutant Metastatic Colorectal Cancer: Safety Lead-In Results From the Phase III BEACON Colorectal Cancer Study. J Clin Oncol \\ 2019;37:1460-9
}

Submitted Aug 14, 2019. Accepted for publication Aug 22, 2019.

doi: 10.21037/atm.2019.08.118

View this article at: http://dx.doi.org/10.21037/atm.2019.08.118

$B R A F$ mutations occur in $8-12 \%$ of metastatic colorectal cancers (mCRC) and more than $95 \%$ of them consist in a $\mathrm{T}>\mathrm{A}$ transversion in exon 15 resulting in an amino acid substitution from valine to glutamic acid (V600E) that is able to increase BRAF kinase activity by approximately 10 -fold $(1,2)$. BRAF V600E mutant $\mathrm{mCRC}$ is a hard challenge of modern oncology due to its awfully poor prognosis: the median overall survival (OS) of patients affected by BRAF mutant mCRC with standard treatments is around 12 months, that is close to the median first-line progression free survival (PFS) of patients with $B R A F$ wildtype tumours $(1,2)$. In fact, not only the clinical outcome with upfront doublets plus a targeted agent is unsatisfactory, but also the percentage of patients able to receive further treatments after progression is extremely low and the benefit provided is limited, with reported overall response rate (ORR) of less than $10 \%$, median PFS of about 2 months, and median OS ranging from 4 to 6 months (3-5).

Therefore, drawing from the rationale of counteracting the intrinsic biologic aggressiveness of this disease with an intensified upfront regimen, and based on results of a retrospective experience (6), a prospective phase II study (7), and a subgroup analysis of a phase III randomized study (8), FOLFOXIRI (fluorouracil, oxaliplatin and irinotecan) plus bevacizumab is now regarded as a "standard" firstline option for patients with $B R A F$ mutant mCRC, able to receive this treatment.

Nevertheless, this recommendation is based on a relatively small number of treated patients and only a percentage of $B R A F$ mutant patients in the clinical practice is fit enough to receive this regimen, also considering the higher incidence of BRAF V600E mutation among elderly patients and the frequent occurrence in patients with suboptimal general conditions (ECOG PS 2).

Many efforts have been made in order to turn $B R A F$ V600E mutation from a strong point for the tumour into its Achille's heal. Initial results with BRAF inhibitors were unexpectedly disappointing compared to those achieved in metastatic melanoma. Only one partial response was reported among 21 patients treated with vemurafenib monotherapy in a phase II study with a median PFS of 2.1 months (9). An explanation to this failure was found moving back from bedside to bench, since preclinical data suggested an hyperactivation of EGFR able to convey a reactivation of MAPKs in CRC cell lines-and not in melanoma lines-following BRAF inhibition. Targeting EGFR was an efficacious strategy to make these cell lines sensitive to the BRAF inhibitor, thus achieving a synergistic inhibition of tumour growth. Subsequent phase I and II studies combining BRAF inhibitors (vemurafenib and dabrafenib) with anti-EGFR monoclonal antibodies (cetuximab or panitumumab) confirmed improved activity in BRAFV600E mutated mCRC, but with heterogeneous results across different trials assessing different combinations (10).

Preclinical studies showed that a deeper inhibition of 
the MAPK pathway could be obtained by combining BRAF and MEK inhibition. Differently from results in advanced melanoma, this strategy as well as other combinations of chemotherapy and BRAF/EGFR inhibitors did not provide the awaited improvements with an ORR of approximately $20 \%$ and a median PFS of $4-5$ months, highlighting the need to develop more effective therapies (11). In this scenario, the triple combination of BRAF, MEK and EGFR inhibitors was evaluated. A phase II study of dabrafenib, trametinib and panitumumab reported an encouraging ORR of $21 \%$, with a not impressive median PFS of 4.1 months (12). More recently, a new ATP-competitive BRAF inhibitor with a more than 10-time longer dissociation half-life ( $>30$ hours) than either dabrafenib or vemurafenib, encorafenib, was developed. The combination of encorafenib with cetuximab showed promising results in a phase II study enrolling patients who had progressed after at least one therapy with a confirmed ORR of $24 \%$, a PFS of 4.2 months and a median OS of 9.3 months (13). The same combination with the addition of the non-ATP-competitive, allosteric MEK1/2 inhibitor binimetinib was evaluated in the BEACON study $(14,15)$. This open-label, randomized, three-arm, phase III trial was launched to evaluate the efficacy and safety of encorafenib plus cetuximab with or without binimetinib versus cetuximab combined with either irinotecan or FOLFIRI at investigators' choice in patients with $B R A F V 600 E$ mutated mCRC progressed after one or two prior regimens. Since the triplet combination of binimetinib, encorafenib and cetuximab had not been clinically evaluated before, a safety lead-in (SLI) phase including around 30 patients was planned to determine the safety, tolerability and preliminary activity of the "biological triplet" at the doses planned for the randomized phase of the trial. Overall, the treatment was well tolerated with a safety profile similar to that previously reported for individual agents, being fatigue (13\%), anaemia (10\%), increased AST (10\%), increased creatine-phosphokinase (10\%) and urinary tract infections $(10 \%)$ the most common grade 3 or 4 adverse events. Grade 3/4 skin toxicities were rare and were less common than the $12 \%$ rate of rash reported with cetuximab monotherapy, suggesting that the simultaneous BRAF inhibition might be able mitigate this cetuximabrelated adverse event. Efficacy results of the SLI phase were quite exciting with a confirmed ORR of $48 \%$, median PFS of 8.0 months and median OS of 15.3 months, almost doubled as compared to other triple combinations (14). Based on these data, NCCN guidelines included this triplet combination as a treatment option in patients with
$B R A F$ mutated mCRC progressed after one or two prior regimens (16).

At the last ESMO World Congress on Gastrointestinal Cancer, the overall results of the phase III BEACON trial were presented (15). In the original design, the primary endpoint of the study was the OS of the triplet versus the control arm. PFS and ORR of triplet versus control and OS, PFS and ORR of doublet versus control would have been tested as secondary endpoints in a hierarchical manner if the primary endpoint was met. Results of the SLI led to the inclusion of ORR as an additional primary endpoint and to the introduction of an interim OS analysis to allow an early assessment of trial's results. Overall, 224 patients were treated with encorafenib, binimetinib and cetuximab, 220 with encorafenib and cetuximab and 221 with irinotecan or FOLFIRI plus cetuximab. The study met its primary endpoints. At a median follow-up of 7.8 months, median OS was significantly longer in the triplet arm respect to the control arm (9.0 vs. 5.4 months; HR: 0.52, 95\% CI: $0.39-0.70 ; \mathrm{P}<0.0001)$ and also ORR was significantly higher in the triplet arm $(26 \%$ vs. $2 \% ; \mathrm{P}<0.0001)$. In addition, all other secondary endpoints were in favour of the triplet or doublet arm as compared with the control arm (Table 1) (15). Notable, even if the study was not powered to formally compare the results of the triplet to the doublet combination, the efficacy and activity of encorafenib, binimetinib and cetuximab was similar to encorafenib and cetuximab. The safety profile of the triplet was acceptable, similar to that of the control arm, but worse than the doublet. Most common grade 3 or 4 AEs of triplet, doublet and control arm reported in more than $5 \%$ were diarrhoea (10\% vs. $2 \%$ vs. $10 \%)$, abdominal pain ( $6 \%$ vs. $2 \%$ vs. $5 \%$ ), nausea $(5 \%$ vs. $<1 \%$ vs. $1 \%)$, asthenia ( $3 \%$ vs. $3 \%$ vs. $5 \%)$ and anaemia (10\% vs. $5 \%$ vs. $4 \%)(15)$.

BEACON is the first phase III trial conducted in the specific population of BRAF V600E-mutant mCRC patients and provides the first demonstration of the efficacy of a targeted approach in this subgroup at poor prognosis. Moreover, the "BEACON strategy" stands as one of the first examples of personalized oncology in the field of colorectal cancer, identifying BRAF V600 mutation as a positive predictor of benefit from a therapeutic approach.

At the same time, both the study design and its findings deserve some considerations.

Firstly, the choice of the control arm of the BEACON study is rather questionable, as the usefulness of antiEGFR antibodies in BRAF mutant mCRC is limited or null, especially in the second and further lines of treatment. 
Table 1 Activity and efficacy results in the BEACON trial

\begin{tabular}{lccc}
\hline $\begin{array}{l}\text { Response } \\
\text { and survival } \\
\text { parameters }\end{array}$ & $\begin{array}{c}\text { Encorafenib }+ \\
\text { binimetinib }+ \\
\text { cetuximab }\end{array}$ & $\begin{array}{c}\text { Encorafenib }+ \\
\text { binimetinib }\end{array}$ & $\begin{array}{c}\text { FOLFIRI or } \\
\text { Irinotecan }+ \\
\text { cetuximab }\end{array}$ \\
\hline $\begin{array}{l}\text { Evaluable for } \\
\text { response (first }\end{array}$ & $\mathrm{N}=111$ & $\mathrm{~N}=113$ & $\mathrm{~N}=107$ \\
$\begin{array}{l}\text { 331 randomized } \\
\text { patients) }\end{array}$ & & & \\
CR & $4 \%$ & $5 \%$ & $0 \%$ \\
PR & $23 \%$ & $15 \%$ & $2 \%$ \\
SD & $42 \%$ & $54 \%$ & $29 \%$ \\
PD & $10 \%$ & $7 \%$ & $34 \%$ \\
Not evaluable \\
by RECIST
\end{tabular}

$\mathrm{N}$, number; CR, complete response; PR, partial response; SD, stable disease; $\mathrm{PD}$, progression disease; ORR, overall response rate; PFS, progression-free survival; ITT, intention to treat; OS, overall survival; $\mathrm{HR}$, hazard ratio; $\mathrm{Cl}$, confidence interval.

The PICCOLO study showed a statistically significant detrimental effect of the addition of anti-EGFR to irinotecan in terms of PFS when administered in the second line therapy of $B R A F$-mutant mCRC (17). On the other hand, an advantage by adding aflibercept or ramucirumab to second-line FOLFIRI was suggested in the VELOUR (18) and RAISE (19) study respectively, with a median OS of 9-10 months with the antiangiogenics as compared with 4-5 months with FOLFIRI alone.

Secondly, even if not formally compared, outcomes reported with the triplet and the doublet are quite similar with a better safety profile for the doublet, thus not demonstrating any reduction of the BRAF inhibitor-related toxicity, especially the occurrence of secondary squamouscell skin cancer and other skin adverse events with the addition of the MEK inhibitor, as previously demonstrated with vemurafenib/cobimetinib (20) and dabrafenib/ trametinib (21). This observation might be explained by the higher extent of BRAF inhibition by encorafenib-as compared with other BRAF inhibitors-that could reduce the paradoxical activation of MAPK pathways in normal tissues, thus limiting adverse events associated with this biological effect, while enhancing the anti-tumour activity. This hypothesis is supported also by the low incidence of skin-related toxicities, and in particular of secondary skin malignancies, reported so far. By a clinical point of view, based on available data, the added value of the MEK inhibitor in this therapeutic strategy is questionable.

Thirdly, an open issue is the management of mCRCs bearing both BRAF V600E mutation and microsatellite instability, considering the association of these molecular characteristics in up to $30 \%$ of $B R A F$ mutant mCRCs (1). Only the $5-10 \%$ of patients included in the BEACON study had a MSI-high or dMMR tumour, probably as a consequence of the simultaneous diffusion of checkpoint inhibitors as a therapeutic tool for MSI-high tumours in the United States. Based on available data, though in the absence of a formal comparison, results achieved with immunotherapy agents seem more convincing and are independent of $B R A F$ mutational status $(22,23)$.

Forthly, emerging evidence shows a high degree of heterogeneity among $B R A F$-mutant mCRC patients both by a clinical and molecular point of view (24). From a molecular perspective, two distinct subgroups of BRAFV600E mutant CRC were recently distinguished based on gene expression profile beyond microsatellite instability: one, named BM1, exhibiting high KRAS/ mTOR/AKT/4EBP1, EMT activation and immune infiltration and the other, named BM2, presenting cell cycle checkpoint dysregulation. BM1 cell lines are more sensitive to BRAF, BCL2 and MEK inhibition as compared with BM2 lines. On the other hand, BM2 cell lines are more sensitive to CDK1 inhibition as compared with BM1. Therefore, a retrospective gene expression analysis of tumour tissues of patients enrolled in the BEACON trial could help to personalize treatment choices in $B R A F$ mutant mCRC patients.

In conclusion, the BEACON study lights up a new 
hope for $B R A F$ mutant patients and for the development of targeted strategies in $\mathrm{mCRC}$. The combination of encorafenib and cetuximab with or without binimetinib should become a new standard in this setting. A phase II study named ANCHOR-CRC (encorAfenib, biNimetinib and Cetuximab in Subjects witH previOusly Untreated BRAF-mutant ColoRectal Cancer) is currently ongoing to explore the usefulness of this approach also in first-line (25).

\section{Acknowledgments}

None.

\section{Footnote}

Conflicts of Interest: C Cremolini reported receiving personal fees from F. Hoffman-La Roche, Bayer, Sirtex, and Amgen. A Falcone reported receiving grants and personal fees from F. Hoffman-La Roche, Amgen, and Merck Serono as well as personal fees from Celgene, Bayer, and Sanofi Aventis. The other authors have no conflicts of interest to declare.

Ethical Statement: The authors are accountable for all aspects of the work in ensuring that questions related to the accuracy or integrity of any part of the work are appropriately investigated and resolved.

\section{References}

1. Venderbosch S, Nagtegaal ID, Maughan TS, et al. Mismatch repair status and BRAF mutation status in metastatic colorectal cancer patients: a pooled analysis of the CAIRO, CAIRO2, COIN, and FOCUS studies. Clin Cancer Res 2014;20:5322-30.

2. Safaee Ardekani G, Jafarnejad SM, Tan L, et al. The prognostic value of BRAF mutation in colorectal cancer and melanoma: a systematic review and meta-analysis. PloS One 2012;7:e47054.

3. Heinemann V, von Weikersthal LF, Decker T, et al. FOLFIRI plus cetuximab versus FOLFIRI plus bevacizumab as first-line treatment for patients with metastatic colorectal cancer (FIRE-3): a randomised, open-label, phase 3 trial. Lancet Oncol 2014;15:1065-75.

4. Schwartzberg LS, Rivera F, Karthaus M, et al. PEAK: a randomized, multicenter phase II study of panitumumab plus modified fluorouracil, leucovorin, and oxaliplatin (mFOLFOX6) or bevacizumab plus mFOLFOX6 in patients with previously untreated, unresectable, wild-type KRAS exon 2 metastatic colorectal cancer. J Clin Oncol 2014;32:2240-7.

5. Morris V, Overman MJ, Jiang ZQ, et al. Progression-free survival remains poor over sequential lines of systemic therapy in patients with BRAF-mutated colorectal cancer. Clin Colorectal Cancer 2014;13:164-71.

6. Masi G, Loupakis F, Salvatore L, et al. Bevacizumab with FOLFOXIRI (irinotecan, oxaliplatin, fluorouracil, and folinate) as first-line treatment for metastatic colorectal cancer: a phase 2 trial. Lancet Oncol 2010;11:845-52.

7. Loupakis F, Cremolini C, Salvatore L, et al. FOLFOXIRI plus bevacizumab as first-line treatment in BRAF mutant metastatic colorectal cancer. Eur J Cancer 2014;50:57-63.

8. Cremolini C, Loupakis F, Antoniotti C, et al. FOLFOXIRI plus bevacizumab versus FOLFIRI plus bevacizumab as first-line treatment of patients with metastatic colorectal cancer: updated overall survival and molecular subgroup analyses of the open-label, phase 3 TRIBE study. Lancet Oncol 2015;16:1306-15.

9. Kopetz S, Desai J, Chan E, et al. Phase II Pilot Study of Vemurafenib in Patients With Metastatic BRAF-Mutated Colorectal Cancer. J Clin Oncol 2015;33:4032-8.

10. Yaeger R, Cercek A, O'Reilly EM, et al. Pilot trial of combined BRAF and EGFR inhibition in BRAF-mutant metastatic colorectal cancer patients. Clin Cancer Res 2015;21:1313-20.

11. Corcoran RB, Atreya CE, Falchook GS, et al. Combined BRAF and MEK Inhibition With Dabrafenib and Trametinib in BRAF V600-Mutant Colorectal Cancer. J Clin Oncol. 2015;33:4023-31.

12. Corcoran RB, André T, Atreya CE, et al. Combined BRAF, EGFR, and MEK Inhibition in Patients with BRAFV600E-Mutant Colorectal Cancer. Cancer Discov 2018;8:428-43.

13. Tabernero J, Geel RV, Guren TK, et al. Phase 2 results: Encorafenib (ENCO) and cetuximab (CETUX) with or without alpelisib (ALP) in patients with advanced BRAFmutant colorectal cancer (BRAFm CRC). J Clin Oncol 2016;34:abstr 3544.

14. Van Cutsem E, Cuyle P-J, Huijberts S, et al. BEACON CRC study safety lead-in (SLI) in patients with BRAFV600E metastatic colorectal cancer (mCRC): Efficacy and tumor markers. J Clin Oncol 2018;36:abstr 627.

15. Kopetz S, Grothey A, Van Cutsem E, et al. LBA006BEACON CRC: a randomized, 3-Arm, phase 3 study of encorafenib and cetuximab with or without binimetinib 
vs. choice of either irinotecan or FOLFIRI plus cetuximab in BRAF V600E-mutant metastatic colorectal cancer. Ann Oncol 2019;30:mdz183.004. Available online: https://doi. org/10.1093/annonc/mdz183.004

16. National Comprehensive Cancer Network Guidelines: Colon Cancer (Version 2.2019). Available online: https:// www.nccn.org/professionals/physician_gls/pdf/colon.pdf

17. Seymour MT, Brown SR, Middleton G, et al.

Panitumumab and irinotecan versus irinotecan alone for patients with KRAS wild-type, fluorouracil-resistant advanced colorectal cancer (PICCOLO): a prospectively stratified randomised trial. Lancet Oncol 2013;14:749-59.

18. Van Cutsem E, Tabernero J, Lakomy R, et al. Addition of aflibercept to fluorouracil, leucovorin, and irinotecan improves survival in a phase III randomized trial in patients with metastatic colorectal cancer previously treated with an oxaliplatin-based regimen. J Clin Oncol 2012;30:3499-506.

19. Tabernero J, Yoshino T, Cohn AL, et al. Ramucirumab versus placebo in combination with second-line FOLFIRI in patients with metastatic colorectal carcinoma that progressed during or after first-line therapy with bevacizumab, oxaliplatin, and a fluoropyrimidine (RAISE): a randomised, double-blind, multicentre, phase 3 study. Lancet Oncol 2015;16:499-508.

20. Larkin J, Ascierto PA, Dréno B, et al. Combined

Cite this article as: Cremolini C, Moretto R, Zucchelli G, Falcone A. BRAF mutant metastatic colorectal cancers: new arrows in our quiver. Ann Transl Med 2019;7(Suppl 8):S367. doi: $10.21037 /$ atm.2019.08.118
Vemurafenib and Cobimetinib in BRAF-Mutated Melanoma. N Engl J Med 2014;371:1867-76.

21. Long GV, Stroyakovskiy D, Gogas H, et al. Combined BRAF and MEK inhibition versus BRAF inhibition alone in melanoma. N Engl J Med 2014;371:1877-88.

22. Overman MJ, McDermott R, Leach JL, et al. Nivolumab in patients with metastatic DNA mismatch repair-deficient or microsatellite instability-high colorectal cancer (CheckMate 142): an open-label, multicentre, phase 2 study. Lancet Oncol 2017;18:1182-91.

23. Overman MJ, Lonardi S, Wong KYM, et al. Durable Clinical Benefit With Nivolumab Plus Ipilimumab in DNA Mismatch Repair-Deficient/Microsatellite Instability-High Metastatic Colorectal Cancer. J Clin Oncol 2018;36:773-9.

24. Loupakis F, Intini R, Cremolini C, et al. A validated prognostic classifier for V600EBRAF-mutated metastatic colorectal cancer: the 'BRAF BeCool' study. Eur J Cancer 2019;118:121-30.

25. Grothey A, Yaeger R, Paez D, et al. P-400ANCHOR CRC: a phase 2, open-label, single arm, multicenter study of encorafenib (ENCO), binimetinib (BINI), plus cetuximab (CETUX) in patients with previously untreated BRAF V600E-mutant metastatic colorectal cancer (mCRC). Ann Oncol 2019;30:mdz155.399. Available online: https://doi. org/10.1093/annonc/mdz155.399 